\title{
Estimation of Hyper-Parameters Based on Logit-Normal Model
}

\section{Zehai ZHAO}

Guizhou University, Guiyang 550025 P. R. China; 495359612@qq.com

Mingzhong JIN

Guizhou Minzu University, Guiyang 550025 P. R. China; $13885196899 @ 163 . c o m$

Minqing GONG, Siwen YANG

Guizhou University, Guiyang 550025 P. R. China

minquing@163.com,352333464@qq.com

ABSTRACT: In this paper, we deduce a range of the hyper-parameters, and use Gauss-Hermite quadrature introduced in reference [1] to approximate the moment equation, estimating the moment estimators by enumeration method. Simulation experiment carried out to study the accuracy of estimates by Monte Carlo integration. Simulation experiment shows that estimating parameters from the moment equation of logitnormal model by enumeration method can get very accurate estimates, and verified the EB estimator is robust. We also use the method to estimate poverty incidences in Spain.

KEYWORD: SAE; Logit-normal model; EB; Enumeration method; Poverty incidence

\section{INTRODUCTION}

The commonly used methods for SAE includes synthetic estimation, composite estimation, empirical best linear unbiased prediction (EBLUP), empirical Bayes (EB) method and hierarchical Bayes (HB) method. Empirical Bayes method and hierarchical Bayes methods are applicable more generally in the sense of handling models for binary and count data.....[1]. Hyper-parameters of EB models can usually be estimated by method of the maximum likelihood (ML) or the moment estimation, but ML method is computationally complex. Several models for EB estimation was given in reference[1], including logit-normal model. The moment equation of paramaters can be approximated by using Gauss-Hermite quadrature first, and then its roots be obtained by using NewtonRaphson iterative algorithm[1]. We proposed a feasible approach in this paper to calculate the hyper-parameters of logit-normal model.

\section{LOGIT-NORMAL MODEL[1]}

Consider a two stage model on the sample observations $y_{i j}$, that is

$$
y_{i j}=0 \text { or } 1, \quad j=1, \cdots, n_{i} ; i=1, \cdots, m .
$$

In the first stage, we assume that the proportion of $i$-th area is

$$
p_{i}=\sum_{j} y_{i j} / \sum_{j} 1 \text {, }
$$

and its sample total is

$$
y_{i}=\sum_{j \in S_{i}} y_{i j},
$$

where $S_{i}$ denotes the sample set of $i$-th area, $n_{i}$ is the sample size,

$y_{i} \sim B\left(n_{i}, p_{i}\right)$, that is,

$$
f\left(y_{i} \mid p_{i}\right)=\left(\begin{array}{c}
n_{i} \\
y_{i}
\end{array}\right) p_{i}^{y_{i}}\left(1-p_{i}\right)^{n_{i}-y_{i}}
$$

In the second stage, assume that $p_{i}$ satisfies the logit-normal model:

$$
\log \operatorname{it}\left(p_{i}\right)=\log \left[p_{i} /\left(1-p_{i}\right)\right] \sim N\left(\mu, \sigma^{2}\right)
$$

According to (2), the $p_{i}$ can be written as:

$$
\begin{aligned}
& p_{i}(a(z))=\exp (a(z)) /(1+\exp (a(z))), \\
& z \sim N(0,1) a(z)=\mu+\sigma z
\end{aligned}
$$

According to (1) and (3), the Bayes estimator is:

$\hat{p}_{i}^{\mathrm{B}}=\frac{E\left[h_{1}(a(z)) \exp \left\{h_{2}\left(y_{i}, a(z)\right)\right\}\right]}{E\left[\exp \left\{h_{2}\left(y_{i}, a(z)\right)\right\}\right]}$

where $\varphi(z)$ is the density of normal $N(0,1)$ distribution, and

$$
\begin{aligned}
& h_{1}(a)=\exp (a) /(1+\exp (a)), \\
& h_{2}\left(y_{i}, a\right)=a y_{i}-n_{i} \ln (1+\exp (a))
\end{aligned}
$$


Formula (4) can be evaluated by simulating samples from $N(0,1)$ or using numerical integration.

\section{ESTIMATION OF PARAMETERS $\mu$ AND $\sigma$}

\subsection{Moment equation for $\mu$ and $\sigma$ [1].}

Equate the weighted sample mean and the weighted sample variance to $p_{1}, \cdots, p_{m}$, which leads to:

$$
\begin{gathered}
E(p)=\sum_{i} \frac{n_{i}}{n_{T}} \hat{p}_{i}=\hat{p}, \\
S_{p}^{2}=\sum_{i} \frac{n_{i}}{n_{T}}\left(\hat{p}_{i}-\hat{p}\right)^{2}, \\
n_{T}=n_{1}+\cdots+n_{m}
\end{gathered}
$$

The expectation of $p^{2}$ is obtained from (6), that is

$$
E\left(p^{2}\right)=[E(p)]^{2}+\operatorname{var}(p)=\hat{p}^{2}+S_{p}^{2}
$$

According to (3), (5), (6) and (7), the moment estimators, $\hat{\mu}$ and $\hat{\sigma}$, are given by

$$
\begin{aligned}
& E\left[h_{1}(\mu+\sigma z)\right]=\hat{p}, \\
& E\left[h_{1}^{2}(\mu+\sigma z)\right]=\hat{p}^{2}+S_{p}^{2}
\end{aligned}
$$

Let $A=1-\hat{p}, \quad B=A^{2}+S_{p}^{2}$,

then equation (8) is equivalent to

$$
\begin{aligned}
& \int_{-\infty}^{+\infty} \frac{1}{1+\exp (\mu+\sigma x)} \varphi(x) \mathrm{d} x=A, \\
& \text { and } \int_{-\infty}^{+\infty} \frac{1}{(1+\exp (\mu+\sigma x))^{2}} \varphi(x) \mathrm{d} x=B
\end{aligned}
$$

where $\varphi(x)$ is the density of the normal $N(0,1)$ distribution.

Since the $\hat{p}$ usually satisfies $\hat{p}<1 / 2$, that is, $A>1 / 2$, we assume that $(\hat{\mu}, \hat{\sigma})$ is the solution of equation (9), then we have the following inequality:

$$
\ln (2+B-3 A)-\hat{\sigma}^{2} / 2<\hat{\mu}<\ln (1 / A-1)
$$

\subsection{Enumeration method to obtain the estimates of $\mu$ and $\sigma$}

Assume that

$$
\begin{aligned}
& a<\hat{\mu}<b, c<\hat{\sigma}<d, \text { let } \\
& \mu_{i}=a+(b-a)\left(i / N_{1}\right), \quad i=1, \cdots, N_{1}, \\
& \sigma_{j}=c+(d-c)\left(j / N_{2}\right), \quad j=1, \cdots, N_{2}, \\
& \boldsymbol{\alpha}=\left(\mu_{1}, \cdots, \mu_{N_{1}}\right)^{T},
\end{aligned}
$$

$$
\boldsymbol{\beta}=\left(\sigma_{1}, \cdots, \sigma_{N_{2}}\right)^{T},
$$

then the $i$-th row and $j$-th column of matrix

$$
\boldsymbol{\alpha} \mathbf{1}_{N_{2}}^{T} \text { and } 1_{N_{1}} \boldsymbol{\beta}^{T}
$$

is corresponding to $\left(\mu_{i}, \sigma_{j}\right)$.

For all $i, j$, and positive number $d_{1}$ and $d_{2}$ which are close to 0, we use the Gauss-Hermite quadrature [1] to approximate the left of equation (9), and pick off $\mu_{i}$ and $\sigma_{j}$ which satisfy the following inequality:

$$
\begin{aligned}
& \left|\int_{-\infty}^{+\infty} \frac{1}{1+\exp (\mu+\sigma x)} \varphi(x) \mathrm{d} x-A\right|<d_{1}, \\
& \left|\int_{-\infty}^{+\infty} \frac{1}{(1+\exp (\mu+\sigma x))^{2}} \varphi(x) \mathrm{d} x-B\right|<d_{2}
\end{aligned}
$$

We use the mean of $\mu$ and $\sigma$ or the intersection of two "straight lines" respectively to be the estimates. Substitute the moment estimators $\hat{\mu}$ and $\hat{\sigma}$ into (3), and use the Gauss-Hermite quadrature or $\mathrm{MC}$ method to get a $\mathrm{EB}$ estimator of $p_{i}$.

\section{SIMULATION EXPERIMENT AND RESULTS ANALYSIS}

Assume that there are 50 small areas with 50 samples, that is, $m=50, n_{i}=50$.

Let the sample total for the $i$-th area be $y_{i}$, and the probability of $y_{i j}=1$ be 0.1 , namely, $y_{i} \sim B(50,0.1)$.

Let $n=20$ for the Gauss-Hermite quadrature. The direct estimator of $p_{i}$ is

$$
\hat{p}_{i}=y_{i} / n_{i} .
$$

We do the simulation experiment by MATLAB.

1) Generate 50 binomially distributed random numbers, and compute $\mathrm{A}$ and $\mathrm{B}$ as follows:

$\mathrm{y}=(7378655416343493315643153$ 44334683764165364751055564 ); $\mathrm{A}=0.9068 ; \mathrm{B}=0.823848$.

2) Assume that $\sigma \in\left(\sigma_{0}, \sigma_{1}\right)$ and select the initial value:

$$
\sigma_{0}=0, \sigma_{1}=1,
$$

the interval of $\mu$ is determined by (10). The intervals of $\mu$ and $\sigma$ are divided into 1000 equal sections, let

$$
d_{1}=d_{2}=10^{-5},
$$

and use the Gauss-Hermite quadrature to 
approximate the left of equation (10), the image for (11) is shown in Figure 1.

Reduce the range of $\mu$ and $\sigma$ :

$\sigma_{0}=0.45, \sigma_{1}=0.55$, and let $d_{1}=d_{2}=5 \times 10^{-6}$,

the image of (11) is shown in Figure 2.

Re-adjust the range of $\mu$ and $\sigma$ again:

$\sigma_{0}=0.45, \quad \sigma_{1}=0.47$.

3) Let $\sigma_{0}=0.45, \sigma_{1}=0.47$,

$\mu_{0}=-2.37, \mu_{1}=-2.35 d_{1}=d_{2}=5 \times 10^{-7}$,

intervals of $\mu$ and $\sigma$ are divided into 1000 equal sections, and select the nodes which satisfy (11), we get the nodes:

$$
x=492,492,491 ; y=561,562,565,
$$

the estimates of $\mu$ and $\sigma$ are calculated as:

$$
\hat{\mu}=-2.36017, \quad \hat{\sigma}=0.46123 \text {. }
$$

We substitute the moment estimators

$$
\hat{\mu}=-2.36017, \hat{\sigma}=0.46123
$$

into the left of following (12) and (13) by generating 100000 normally distributed random numbers

$$
x_{1}, \cdots, x_{100000},
$$

we get 6 groups of values as shown in Table 1 .

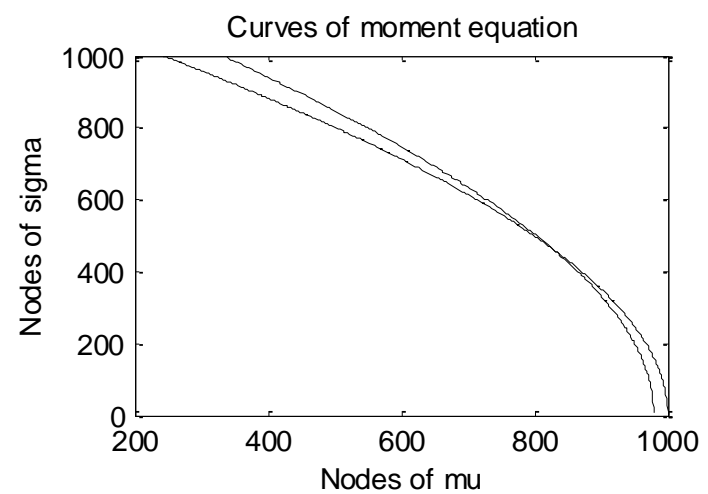

Figure 1. Curves of parameters $\sigma_{0}=0 \quad \sigma_{1}=1$.

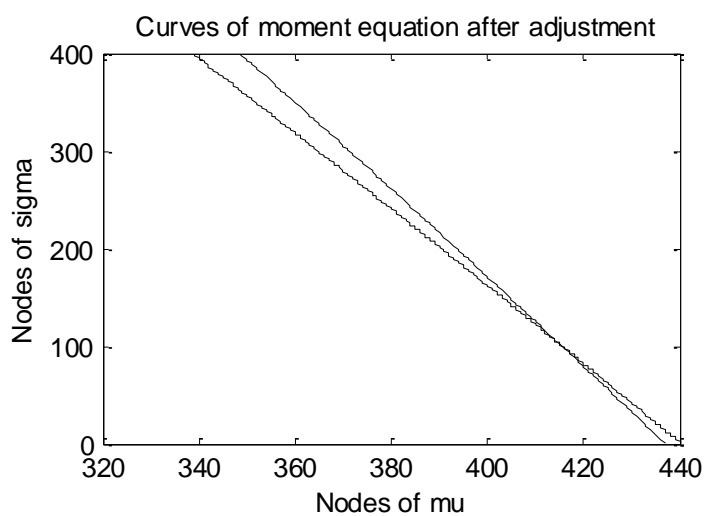

Figure 2. Curves of parameters $\sigma_{0}=0.45 \sigma_{1}=0.55$.

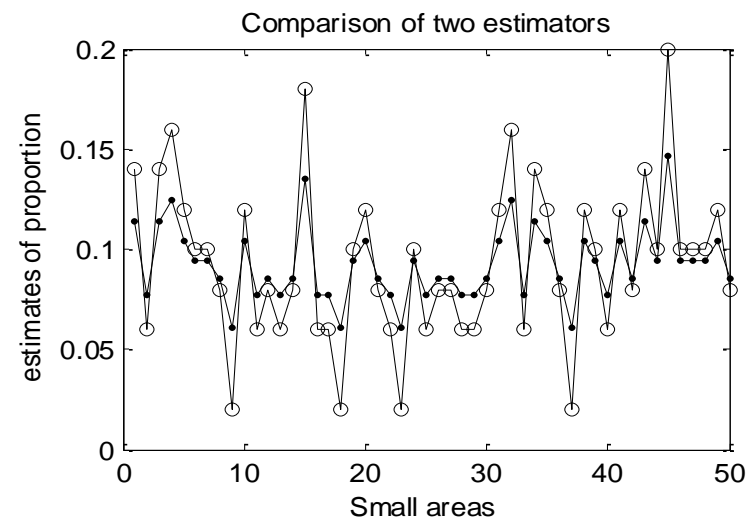

Figure 3. Comparison of two estimators.

$$
\begin{aligned}
& \frac{1}{200000}\left(\sum_{i=1}^{100000} \frac{1}{1+\exp \left(\hat{\mu}+\hat{\alpha} x_{i}\right)}+\frac{1}{1+\exp \left(\hat{\mu}-\hat{\alpha} x_{i}\right)}\right)-A \\
& \frac{1}{200000}\left(\sum_{i=1}^{100000} \frac{1}{\left(1+\exp \left(\hat{\mu}+\hat{\sigma} x_{i}\right)\right)^{2}}+\frac{1}{\left(1+\exp \left(\hat{\mu}-\hat{\sigma} x_{i}\right)\right)^{2}}\right)-B
\end{aligned}
$$

We substitute the moment estimators

$$
\hat{\mu}=-2.36017, \hat{\sigma}=0.46123
$$

into (3), and use the Gauss-Hermite quadrature to get the EB estimator of $p_{i}$.The scatter diagram of $\hat{p}_{i}$ and $\hat{p}_{i ; P E B}$ is shown in Figure 3('o' corresponds to direct estimates, '.' corresponds to EB estimates).

Table 1. Values of formula (12) and (13). unit: $10^{-5}$

\begin{tabular}{|l|l|l|l|l|l|l|}
\hline Value of (12) & 3.10 & -0.12 & -1.23 & -0.08 & -1.25 & 2.90 \\
\hline Value of (13) & 4.71 & -0.09 & -1.84 & -0.29 & -1.88 & 4.59 \\
\hline
\end{tabular}

\section{EXAMPLE}

The EB method based on logit-normal is applied to compute poverty incidences in Spanish provinces. The data of $n_{d}$ and $\hat{p}^{\omega}$ come from the European Survey on Income and Living Conditions for the year 2006[2], we let the $y_{d}$ is close to $n_{d} \cdot \hat{p}^{\omega}$, and get $y_{d}$ to calculate the $\hat{p}^{\mathrm{EB}}$, which is shown in Table 2 .

Table 2. Direct and EB estimates of poverty incidence.

\begin{tabular}{|c|c|c|c|c|}
\hline Province & $n_{d}$ & $y_{d}$ & $\hat{p}^{\omega}(\%)$ & $\hat{p}^{E B}(\%)$ \\
\hline Soria & 17 & 10 & 60.41 & 45.34 \\
\hline Tarragona & 129 & 16 & 12.46 & 12.66 \\
\hline Cordoba & 230 & 71 & 30.66 & 30.38 \\
\hline Badajoz & 427 & 173 & 36.58 & 36.31 \\
\hline Barcelona & 1482 & 161 & 10.82 & 10.91 \\
\hline
\end{tabular}

The estimates of $\mu$ and $\sigma$ are calculated as: 
$\hat{\mu}=-1.66964 ; \hat{\sigma}=0.80218$.

Substitute the moment estimators $\hat{\mu}$ and $\hat{\sigma}$ into (3), and use MC integration as in part 4 to get the EB estimates of $p_{i}$ as shown in Table 2. Values of $\hat{p}^{\mathrm{EB}}$ in Table 2 show that the estimates is adjusted to a reasonable "line" by using EB method, especially for the small sample size. And the EB estimators are closer to direct estimators with the sample size increasing.

\section{SUMMARY}

By Monte Carlo method we have: It would be an efficient numerical method to use Gauss-Hermite quadrature to approximate the moment equation of logit-normal model .It would be easy to find a short interval of $\mu$ and $\sigma$ by observing the image of parameters and estimate the hyper-parameters by using enumeration method; The EB estimators based on logit-nomal model is robust. 4). Estimation of proportion for rare events may be not feasible because the curves of moment equation is almost coincident. In this paper inspired by [2] we use different EB method from [2] to calculate the real data example in [2] and our way is shown feasible also.

\section{ACKNOWLEDGMENTS}

National Statistics Bureau of China (major program 2012LZ054). Guizhou Provincial Governor Funding (QianKeJiaoBan[2010]-04). Guiyang Bureau of Sci. and Tech. (ZhuKeHeTong[2011101]1-32 ). Guizhou Dept. of Sci. and Tech. (QianKeHeJiZi[2009]4002).

\section{REFERENCES}

[1] J.N.K.RAO. Small Area Estimation. American: Wiley, 2003: 197-201.

[2] Isable Molina and J.N.K.RAO. Small Area Estimation of Poverty Indicators. The Canadian Journal of Statistics, 2010(38):369-385.

[3] Yongjin Jin. Sampling Survey: Theory and Application. China: Higher Education Press.

[4] Christian P.Robert. Monte Carlo Methods. Springer Tests in Statistics.

[5] Danny Pfeffermann. New Important Developments in Small Area Estimation. Statistical Science, 2013, Vol.28 (No.1).

[6] L.Wei, W. Zhang. Bayesian Analysis. Press of University of Science and Technology of China 